\title{
A pilot study evaluating the utility of a novel tube cricothyrotomy technique in providing ventilation in small animals using a live porcine model
}

This article was published in the following Dove Press journal: Veterinary Medicine: Research and Reports

\section{Sureiyan Hardjo \\ Catriona Croton \\ Mark D Haworth}

School of Veterinary Science, The University of Queensland, Gatton 4343, Queensland, Australia
Correspondence: Sureiyan Hardjo School of Veterinary Science, The University of Queensland, Gatton 4343, Queensland, Australia

Emails.hardjo@uq.edu.au
Background: Tube cricothyrotomy has been suggested as a first-choice for emergency surgical airway access in small animals, rather than the more commonly accepted procedures of tracheotomy and needle cricothyrotomy.

Methods: A small pilot study of tube cricothyrotomy in a live porcine model was conducted to evaluate this method. The technique was modified to permit the use of commonly available equipment and ease of application. Following ethics approval, a tube cricothyrotomy was performed on 9 live pigs. The endpoints were the efficacy of ventilation through the tube as measured by end-tidal carbon dioxide, successful placement of the tube and the time taken for placement.

Results: Of the nine pigs, eight had a tube placed successfully, with the median procedure time being 111.5 seconds (range: 35-240 seconds). After 10 mins, the mean end-tidal carbon dioxide was $58.3 \mathrm{mmHg}$ (95\% CI: $55.2-61.3 \mathrm{mmHg}$ ), with a range of 54-64 $\mathrm{mmHg}$. Tube cricothyrotomy holds promise as a means of rapidly obtaining tracheal access in small animals and temporarily sustaining ventilation in an emergency.

Conclusion: Further detailed investigation is warranted to assess the use of this technique in the small animal emergency setting.

Keywords: cricothyrotomy, tracheostomy, canine, ventilation, airway, obstruction, front of neck access

\section{Introduction}

Upper airway obstruction is a life-threatening emergency that requires airway access to be re-established rapidly in order to supply oxygen and allow ventilation. An emergency tracheotomy (TT) has been accepted as the treatment of choice in small animals in a "cannot intubate, cannot oxygenate" situation (CICO). ${ }^{1,2}$ Another option is needle cricothyrotomy (CTT) with a large-bore catheter, but this is considered a last resort to supply oxygen as ventilation is either inadequate or relatively complex equipment is required to facilitate it. ${ }^{3,4}$

Tube cricothyrotomy, also known as surgical CTT, has now been suggested as a first-choice method for emergency surgical airway access in small animals. ${ }^{5}$ This is an attractive alternative due to the relative speed of the procedure, reduced complexity when compared with a $\mathrm{TT}^{6}$ and an improved ability to ventilate when compared to needle CTT. Human studies have advocated its use in patients with challenging neck anatomy, while also demonstrating a lack of significant complications when directly compared with TT. ${ }^{6}$ Although there is some debate about which 
technique is superior, in human medicine CTT remains "the fastest and most reliable method of securing the airway in the emergency setting", 7,8

Rapidity of placement is key in emergent settings and a time limit of between 180 and 300 seconds has been suggested as acceptable for a CTT procedure. ${ }^{9}$ Hansen and Eriksen described a method for tube CTT in dog and cat cadavers which they stated was a "feasible, technically simple, safe and rapid procedure for both acute emergency and elective airway access". Several studies examining dogs as experimental models for placement of tube CTT in human medicine have been performed. ${ }^{10-12}$ One of which studied 40 dogs having tubes in place for 14-42 days, resulted in no signs of long-term complications such as stenosis or cricoid cartilage necrosis. ${ }^{11}$ However, to the authors' knowledge, there have been no in vivo trials on the implementation and efficacy of ventilation through tube CTT used in small animals in the face of an upper airway obstruction.

This study seeks to provide information on an alternative airway management strategy in the emergent setting. The authors present a modification to a previously described CTT method $^{5}$ using common equipment available in most veterinary practices. The primary aim of this study was to demonstrate evidence for tube CTT's in vivo efficacy in facilitating ventilation in live pigs as an experimental model for small animals using this modified technique. A secondary aim was to evaluate the success of tube placement in a simulated emergency setting. The final aim was to determine if reestablishment of an airway could be achieved within the accepted limit of $180-300$ seconds.

\section{Materials and methods}

This study was approved by The University of Queensland's Animal Ethics committee (approval number SVS/146/18), which follows the Australian code for the care and use of animals for scientific purposes.

Weaner domestic pigs were chosen as a model due to their body size to mirror the effects of smaller diameter airways encountered with many companion animals. Resistance to airflow increases exponentially with decreases in diameter of endotracheal tubes. ${ }^{13,14}$ As tube size is dictated by patient size, smaller animals may experience a relatively larger impact in reduced airflow and ventilation through small CTT tubes. As increasing tube length also adds resistance to flow, all tubes were cut to an equal length $(70 \mathrm{~mm})$.

Nine pigs were sourced from a previously approved nonrecovery castration surgery practical for veterinary students, which were all scheduled for euthanasia under general anesthesia. The number of pigs was governed by availability on the study day. All pigs were given analgesia and a premedication of $0.3 \mathrm{mg} / \mathrm{kg}$ of methadone and $4 \mathrm{mg} / \mathrm{kg}$ zolazepam/tiletamine intramuscularly. Two $\mathrm{mg} / \mathrm{kg}$ of alphaxalone was targeted for anesthetic induction; however, this was dosed to effect to allow intubation. The surgery practical was completed with the pigs maintained under general anesthesia with inhalational isoflurane at $1-2.5 \%$ depending on patient requirements and $100 \%$ oxygen via an orotracheal tube and an out-of-circuit vaporizer. The anesthesia was supervised by a veterinarian familiar with the study protocols.

Equipment required for the tube CTT and airway obstruction procedure is shown in Figure 1. This includes a $3.5 \mathrm{~mm}$ or $4.0 \mathrm{~mm}$ internal diameter uncuffed endotracheal tube cut to $70 \mathrm{~mm}$ in length, a $2.6 \mathrm{~mm}$ external diameter rigid dog urinary catheter, a size 11 scalpel blade with handle and umbilical tape to secure the tube once placed. A tightly wound wad of gauze swabs, blu tack $^{\mathrm{TM}}$, adhesive tape and laryngoscope was required for upper airway obstruction. To prepare for the procedure, the urinary catheter was placed within the lumen of the endotracheal tube to act as a bougie. A tube approximately half the size of that required for endotracheal intubation was used for this procedure as previously recommended. ${ }^{5}$

The CTT procedure commenced immediately following the castration surgery practical. The pig was maintained in dorsal recumbency and the neck was extended by placing a towel below the dorsocaudal cervical region. Inhalant anesthesia was discontinued, and the endotracheal tube was removed. Anesthesia was maintained with intravenous alphaxalone while the pigs were extubated. If reduced depth of anesthesia was noted by indicators of eye position change, response to withdrawal reflex, increased jaw tone, changes in heart rate (HR), respiratory rate (RR) or

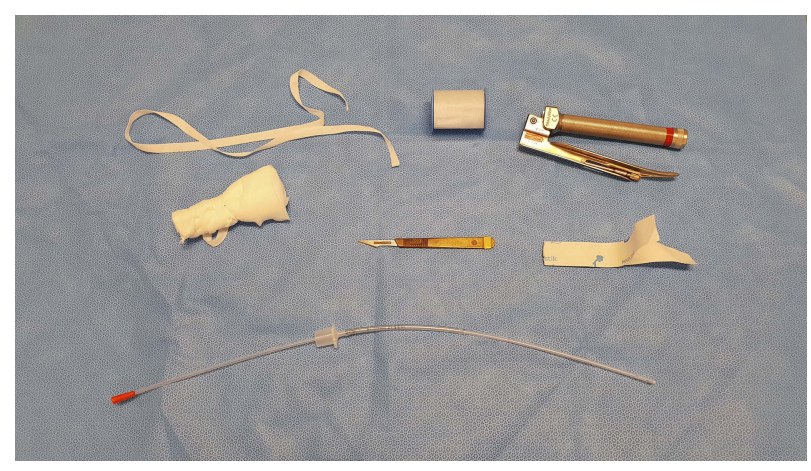

Figure I Equipment required to perform tube CTT using the modified technique (with airway occlusion). 
spontaneous movement, alphaxalone was administered to effect in boluses of approximately $0.5 \mathrm{mg} / \mathrm{kg}$.

To commence the procedure, the veterinarian stood to the right of the patient and identified the cricothyroid notch. To identify the surface landmarks, a modified and abbreviated "laryngeal handshake" was performed. Figure 2 shows a sagittal section of the anatomical structures targeted with the laryngeal handshake. The fingers of the left hand are placed on the cranioventral neck and walked caudally on the midline until the first firm structure is palpated, which is the thyroid cartilage. The thyroid cartilage is then grasped with the left hand (Figure 3A). The index finger is walked along the ventral surface of the thyroid cartilage and the dip in the soft tissue where the thyroid cartilage ends, and cricoid cartilage begins is identified as the cricothyroid notch (Figure 3B). A size 11 scalpel blade was used to make a $20-\mathrm{mm}$, sagittal incision through the skin and subcutaneous tissues over the cricothyroid notch with the right hand (Figure 3C). The same blade was used to make a stab incision through the cricothyroid membrane (Figure 3D).

The stab incision in the cricothyroid membrane was then extended caudally until slight resistance was felt when approaching the cricoid cartilage. With the scalpel blade still in situ and pushing laterally with the flat surface of the blade, the tip of the urinary catheter was inserted immediately into the incision (Figure 3D). It was then angled dorsocaudally and passed into the tracheal lumen for approximately 5-10 cm (Figure 3E). The 70-mm tube was then advanced down the urinary catheter until completely within the trachea and the catheter was removed (Figure $3 \mathrm{~F}$ and $\mathrm{G}$ ). Note, the same procedure may be mirrored for left-hand dominant people. Placement was confirmed with capnometry and the tube was secured with tape around the neck. If the initial approach failed, a wider approach was used (Figure 4). The anesthetic circuit used previously was reconnected and isoflurane anesthesia recommenced. The vaporizer setting was varied using a non-rebreathing circuit in order to maintain general anesthesia. Intermittent boluses of alphaxalone were used as a stopgap to maintain general anesthesia until sufficient depth was achieved.

Following placement of the CTT tube, upper airway obstruction was simulated by placing a wad of gauze swabs in the pharynx, taping the mandible shut and occluding nares with malleable adhesive (blu tack ${ }^{\mathrm{TM}}$ ). This obstruction technique is comparable to that used in a similar study of cannula CTT in dogs. ${ }^{15}$ The obstruction was planned to follow CTT tube placement in case of a prolonged procedure time. This allowed the best chance to facilitate the primary aim of assessing ventilation.

The pigs were monitored for 10 mins post-surgical cricothyrotomy, with recording of end tidal $\mathrm{CO}_{2}\left(\mathrm{ETCO}_{2}\right)$,

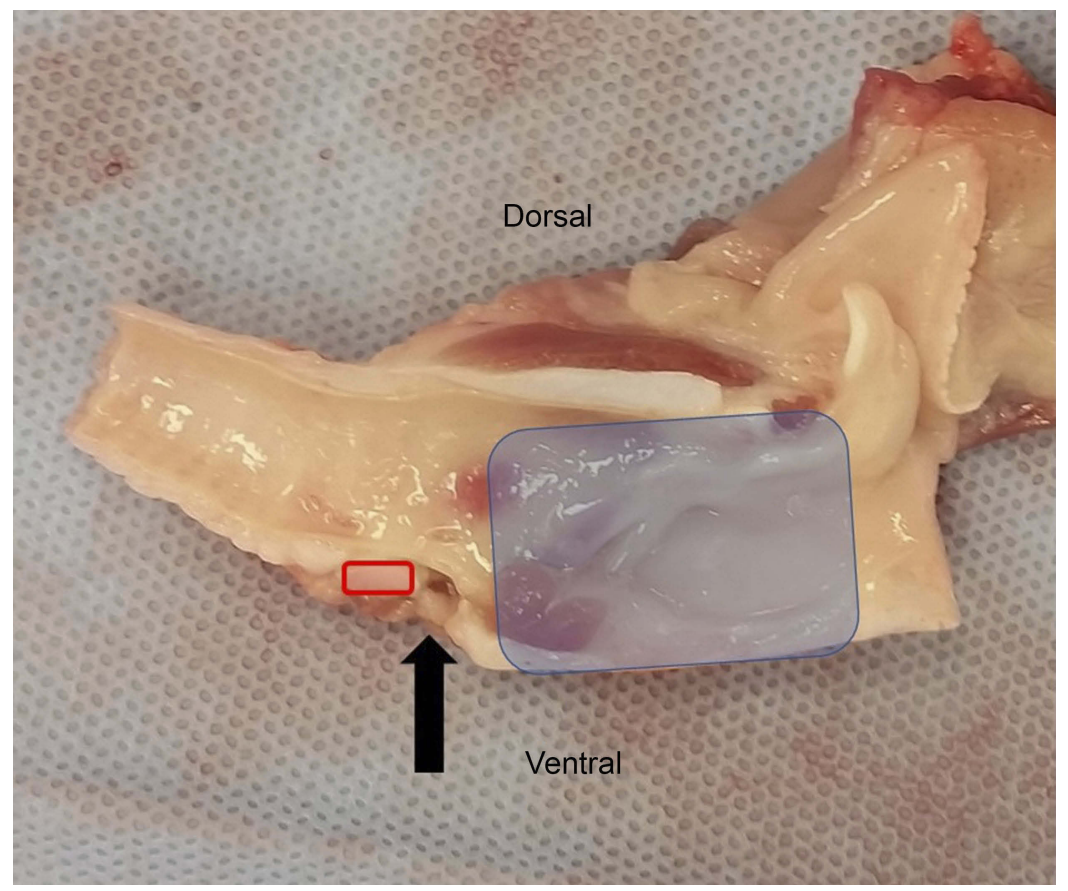

Figure 2 Sagittal section of weaner pig larynx. Blue box indicates thyroid cartilage. Red box indicates cross-section of ventral cricoid cartilage and black arrow indicates the position of the cricothyroid notch. 


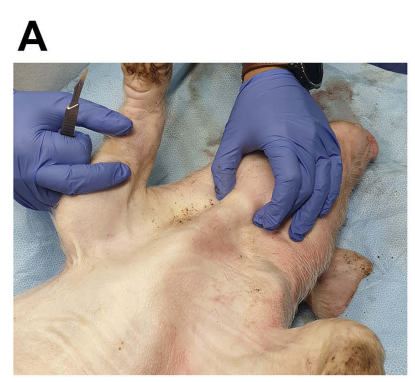

D

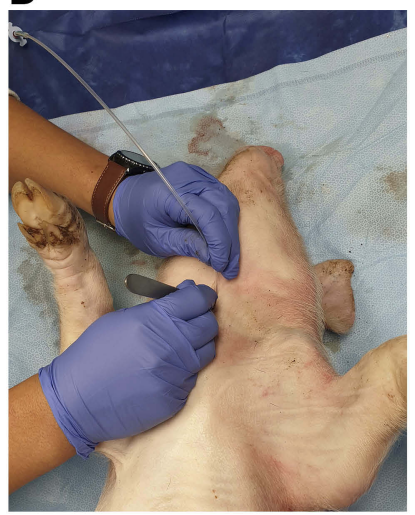

G

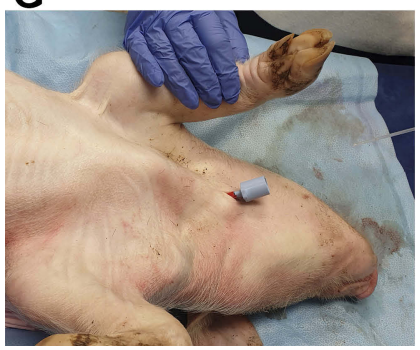

B

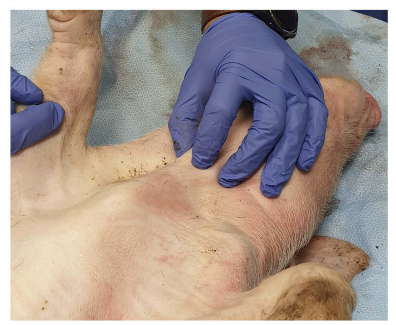

E

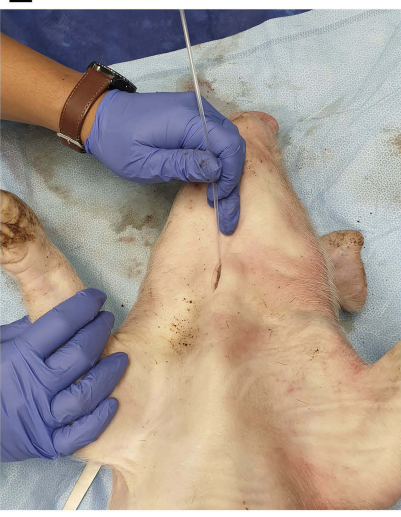

C

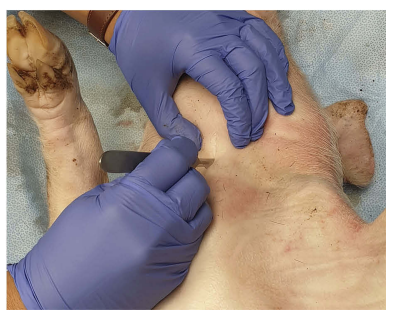

$\mathbf{F}$

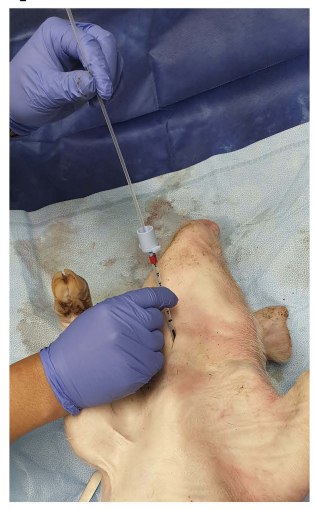

Figure 3 The CTT procedure performed on the pig model (A-G).

A

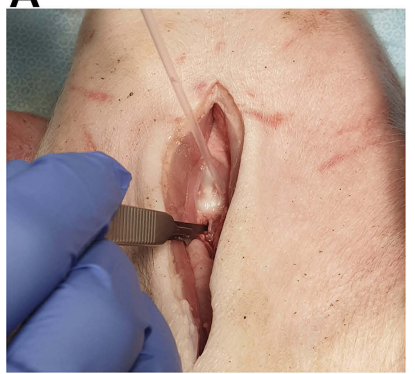

B

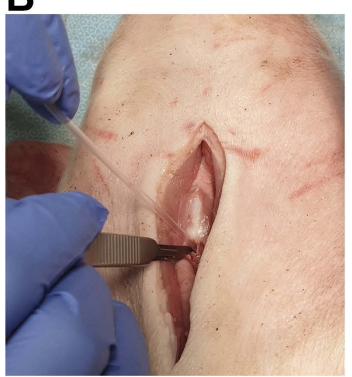

C

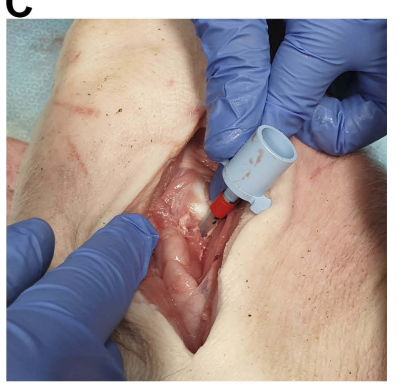

Figure $4 \mathrm{~A}$ dissected view and example of a wide approach of the CTT procedure. The cricothyroid ligament is incised (A). Urinary catheter is placed (B). A size 3.5 uncuffed endotracheal tube is placed into the incision (C).

pulse oximetry $\left(\mathrm{SpO}_{2}\right), \mathrm{HR}$ and RR every minute starting at 1 min post-procedure. Ten minutes was chosen as it is likely to allow time for definitive removal of foreign body obstruction, planning alternate airway access or calling for assistance. Measurements were made with a Bionet BM7 Vet (Tustin, CA). Manual intermittent positive pressure ventilation was performed by squeezing of the reservoir bag on the anesthetic circuit with the adjustable pressure limiting valve closed. It was initiated if the patient was apneic or if $\mathrm{ETCO}_{2}$ readings approached $60 \mathrm{mmHg}$.

Investigators $\mathrm{SH}$ and $\mathrm{MH}$ examined and practiced the intended methodology on a cadaver pig and a cadaver dog 
before commencing the study. All CTT tube placement procedures were performed by one investigator (SH). Timing of the procedure commenced at first incision of the skin and was completed when a typical $\mathrm{ETCO}_{2}$ waveform was confirmed on capnography, indicating correct positioning. If there was sizeable surgical exposure, the skin around the neck was pinched together around the tube to ensure all ventilation occurred through the CTT tube. The $\mathrm{ETCO}_{2}$ was reported as a mean with $95 \%$ confidence interval using a $t$ distribution with 6 degrees of freedom and the time taken for the procedure as a median. Analyses were conducted using Stata Version 14 for Windows (StataCorp. 2015. Statistical Software: Release 14.0. College Station, TX: Stata Corporation).

\section{Results}

The 9 pigs which underwent the procedure are listed in Table 1 in the chronological order in which the CTTs were performed. Successful placement of the cricothyrotomy tube was achieved in 8 of 9 pigs. The first pig had an extra-tracheal placement of the tube, ventral to the trachea, in the subcutis. An initial capnograph reading leads the investigators to believe the procedure was successful and the airway was subsequently obstructed. It was quickly noted from the vital signs that this was not the case and the pig was immediately euthanized under general anesthesia. Pigs 2 through 6 required additional exposure as the tube could not be placed successfully after the first stab incision. The $7^{\text {th }}$ pig had a confirmed tube placement and was stable throughout the allotted 10-minute interval, but the measured data after the first reading was lost. Pig 8 had the tube placed in one attempt; however, the time was longer due to human error, where the urinary catheter/ tube apparatus slipped out of the operator's hand, just before placement.

Seven of the 8 pigs with complete records were spontaneously ventilating before the procedure and 5 of them were spontaneously ventilating at the end of the 10-mins period.

The time-course of $\mathrm{ETCO}_{2}$ changes can be seen for each of the 7 pigs with successful placement of the cricothyrotomy tube and data recorded (Figure 5). This depicts the changes in $\mathrm{ETCO}_{2}$ from immediately post placement of the cricothyrotomy tube and over the following 10 mins. Five of 7 pigs showed a downward trend in their $\mathrm{ETCO}_{2}$. The remaining 2 pigs showed an upward trend, starting with an $\mathrm{ETCO}_{2}$ of 48 and $54 \mathrm{mmHg}$, which increased to 58 and $64 \mathrm{mmHg}$, respectively, with $64 \mathrm{~mm}$

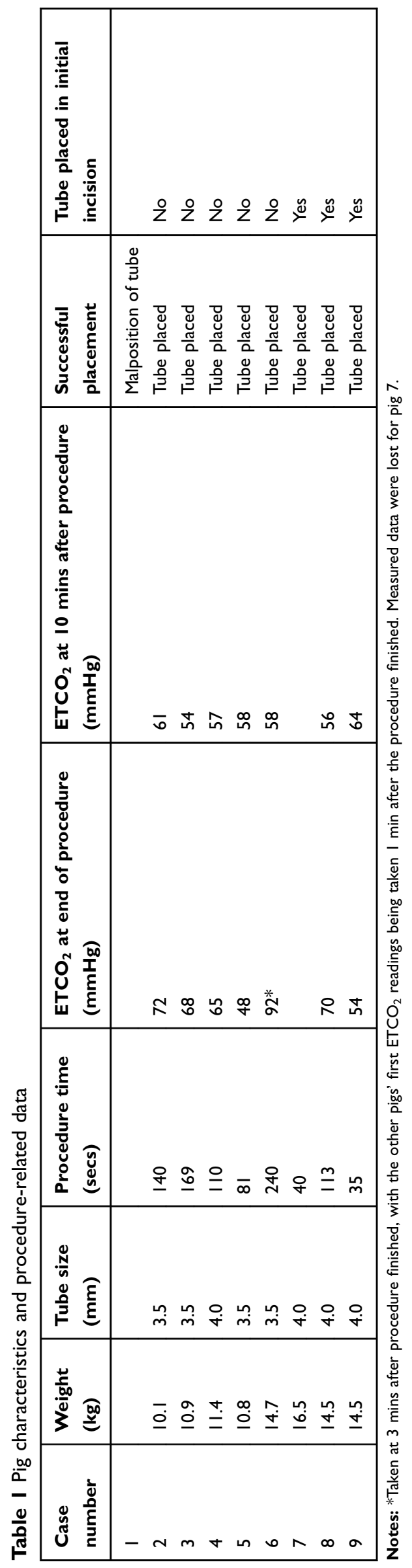




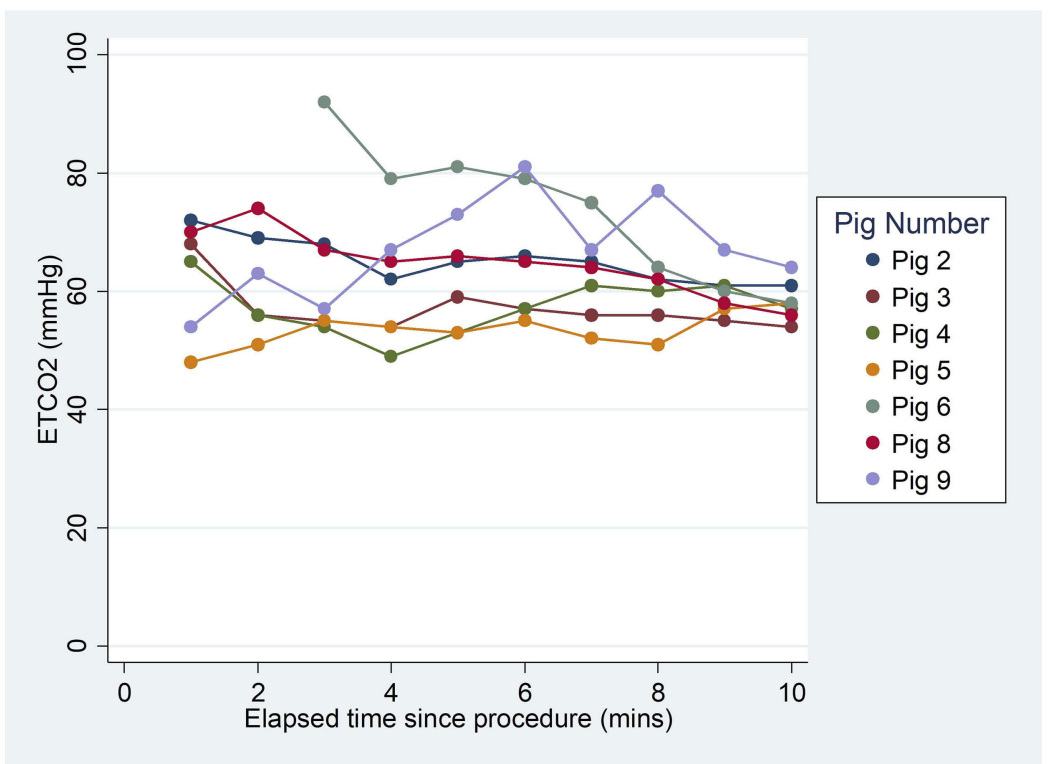

Figure 5 Time-course of changes in $\mathrm{ETCO}_{2}$ for the 10 mins following completion of the procedure.

$\mathrm{Hg}$ the highest $\mathrm{ETCO}_{2}$ recorded after 10 mins. The mean $\mathrm{ETCO}_{2}$ at 10 mins post-procedure for all pigs was 58.3 mmHg (95\% CI: $55.2-61.3 \mathrm{mmHg}$ ). The median time taken to successfully place the CTT tube was $111.5 \mathrm{sec}-$ onds, with the fastest time being 35 seconds and the slowest 240 seconds.

\section{Discussion}

The primary objective of this pilot study was to determine if adequate ventilation was possible via a CTT tube in live weaner pigs using equipment commonly available to the veterinary emergency or general practitioner. It also sought to present a modification to previously described techniques and report ease of placement and procedure time in a simulated small animal emergency setting. This pilot study demonstrated CTT tube placement in live weaner pigs allowed for sufficient ventilation achieving an overall mean $\mathrm{ETCO}_{2}$ of $58.3 \mathrm{~mm} \mathrm{Hg}$ for all pigs. It further demonstrated successful CTT tube placement was also achievable in $88 \%$ of attempts in a simulated emergency setting, with all procedures performed in 4 mins or less.

Upper airway obstruction causing a "cannot intubate, cannot oxygenate" (CICO) situation is often fatal unless rapidly treated. There are several possible causes for upper airway obstruction in small animals including brachycephalic airway disease, neuropathies such as laryngeal paralysis, abscess, hematoma, foreign bodies, trauma and neoplasia. ${ }^{16}$ The currently accepted techniques for gaining surgical airway access in the veterinary literature are TT and needle CTT, ${ }^{1,2}$ although in human medicine, surgical or tube CTT is accepted as a first-choice standard. ${ }^{7,17}$

In recent years there has been debate surrounding the best technique to use in a CICO situation in human medicine. ${ }^{17-19}$ It is generally accepted however that it is of paramount importance to have a simple, safe and rapid procedure. This procedure would also protect against aspiration and provide adequate oxygenation and ventilation. ${ }^{17}$ In the veterinary setting where a CICO emergency may present to a practitioner of any level of experience, it would also ideally use commonly available equipment. Emergency CTT is a technique that is uncommonly performed, even in human medicine,, 20 and it is expected that few veterinarians would be willing to purchase specialized equipment. Furthermore, size range of veterinary patients would mean stocks of multiple kit sizes would need to be maintained, which is not economically effective for low-use equipment.

Hypoventilation as a result of airway obstruction or small diameter tube will lead to hypercapnea. An arterial partial pressure of $\mathrm{CO}_{2} 60 \mathrm{mmHg}$ is an acceptable limit prior to the pathophysiological effects of hypercapnoea. ${ }^{1}$ This limit was adopted as a proxy for effective ventilation. An end-tidal value was used rather than the partial pressure of arterial $\mathrm{CO}_{2}\left(\mathrm{PaCO}_{2}\right)$ due to practical considerations in the student laboratory session. Furthermore, the authors wanted to ensure adequate hypercapneic stimulation for spontaneous ventilation in the face of anesthesia, which may not have occurred if the pigs were manually ventilated to a lower limit. 
The results demonstrate a tube CTT utilizing this modified procedure is compatible with life during an emergent upper airway obstruction and facilitates ventilation. The highest $\mathrm{ETCO}_{2}$ measured at 10 mins from all the pigs was $64 \mathrm{mmHg}$. Only two pigs completed the 10-mins period above $60 \mathrm{mmHg}$. Although pig 5 had an upward trend, it spontaneously ventilated throughout the 10-mins period and had an $\mathrm{ETCO}_{2}$ of $58 \mathrm{mmHg}$ upon completion. This pig achieved an $\mathrm{ETCO}_{2}$ of $51 \mathrm{mmHg}$ at 8 mins post the procedure, indicating that it was not a steady upward trend. Pig 6 had the longest procedure time of all at 240 seconds and as a result, had the highest initial recorded $\mathrm{ETCO}_{2}$ of $92 \mathrm{~mm} \mathrm{Hg}$. A subsequent decrease to a physiologically sustainable value of $58 \mathrm{mmHg}$ with intermittent positive pressure ventilation was able to be achieved. These findings indicated that ventilation through the cricothyrotomy tube was adequate, and the severity of the $\mathrm{ETCO}_{2}$ in this pig was due to the comparative delay in CTT tube placement.

The secondary objective was to assess the clinical utility and success rate of this modified tube CTT procedure in a simulated emergent setting. Successful placement of the modified tube CTT was successful in 8 of 9 pigs $(88 \%)$. Although there are no veterinary data, the success rate of needle CTT in humans has been reported as low as $37 \%,{ }^{21}$ therefore, this appears to be a reasonable alternative. Failed placement in the first pig was likely due to limited experience performing the procedure and interpreting the signs of correct placement. This further highlights the importance of experience and training for emergency airway procedures cited in the literature. ${ }^{22,23}$ Cadaverbased training is superior to simulation in finding surface landmarks for CTT. ${ }^{24}$ Hence, the authors recommend that cadaver labs will be the best training tool to teach the technique to students and veterinarians.

The "laryngeal handshake" as described for humans firstly involves identifying the hyoid bone followed by the thyroid cartilage and then stabilizing the larynx by grasping the cricoid cartilage itself. ${ }^{7,8}$ The ventral cricoid cartilage may be a difficult structure to grasp in smaller or obese animals by an inexperienced veterinarian. Hence, the technique was modified as the thyroid cartilage may provide a larger surface area for clearer identification of landmarks and stabilization of the larynx. Furthermore, after the larynx is released, it allows the left hand free for bougie introduction while the scalpel blade is providing retraction in situ. The procedure in humans requires swapping the scalpel to the left hand as the surgeon stands on the patient's left side. To the authors' knowledge, this method for cricothyroid membrane identification and airway access has not previously been described in the veterinary or human literature and is yet to be validated.

The authors initially followed a "one-step" tube insertion technique ${ }^{5}$ without the urinary catheter in cadaver pigs but were often unable to place the CTT tube correctly as difficulty was encountered pushing through the thick subcutaneous tissue and sternohyoideus muscle. This procedure was easily performed in greyhound cadavers with no subcutaneous fat and a superficial larynx. The difficulties encountered with pigs may indicate the one-step technique may not be appropriate for animals with shorter necks and generous subcutaneous adipose tissue, such as brachycephalic breeds. If a second approach was required for any reason, the initial tract was also lost in the subcutaneous tissues. To address these issues, the authors modified the one-step technique using a urinary catheter as a guide, preplaced in the lumen of the CTT tube (similar to the scalpel-bougie technique used in humans). ${ }^{8}$ Its smaller diameter allowed it to penetrate the subcutis and enter the incision in the cricothyroid ligament easily. The CTT tube was then advanced over the urinary catheter into the trachea. The catheter can be left in situ allowing the tract to remain patent if a different tube is required. The original tube can then be withdrawn and another tube passed over it into the trachea. Note, the luer connector would need to be cut off with smaller diameter tubes.

Rigid dog urinary catheters are widely available in veterinary general practice whereas bougie introducers are not. Benefits of rigid urinary catheters are smaller diameter and relative stiffness which prevent their inadvertent laceration by the scalpel. Laceration of a rubber bougie introducer and subsequent locking to the tube is a known complication in performing CTT termed "bougie trapping". ${ }^{25}$ Even with the most difficult CTTs performed with our technique, this complication did not occur with the same urinary catheter used for all 9 procedures. A further benefit of the urinary catheter is that if the placement of the tip within the trachea is uncertain, a syringe may be attached to the urinary catheter and plunger withdrawn. Loss of plunger resistance confirms that air has been aspirated and the placement in the airway is correct.

A major difference with our technique when compared with the human guidelines is that we propose a vertical incision in the cricothyroid membrane to allow a simpler approach when working with small larynges. A small blood vessel in the cranial portion of the cricothyroid 
ligament (cricothyroid artery) is present in some humans; hence, a horizontal incision, rotation of the blade and second vertical incision caudally is recommended to avoid hemorrhage. ${ }^{7}$ The transverse orientation of this artery and anastomoses with the contralateral side is not described in the $\operatorname{dog}^{26}$ (see Figure 6 for a depiction of the canine cricothyroid ligament with no obvious blood supply or visible cricothyroid artery). Furthermore, a common approach to the larynx in dogs, the ventral laryngotomy, involves incising the entire distance from the cranial thyroid cartilage through to the cricoid cartilage with no mention of hemorrhage as a complication or the requirement to avoid this vessel. ${ }^{27-29}$ This approach even recommends incising through the cricoid cartilage itself in smaller patients. ${ }^{27}$ Although incising the cricoid, termed the "cricoid split", would facilitate introduction of larger tube sizes, we do not recommend it at this stage due to the potential complication of subglottic stenosis.

A benefit of performing this study in a live porcine model was that hemorrhage was able to be assessed and at no time during any procedure was hemostasis required.

One study has shown similar laryngeal dimensions between adult humans and adult dogs weighing an average of $23 \mathrm{~kg} .{ }^{30,31}$ The average "anterior gap between thyroid and cricoid cartilages" of these dogs exceeded that of human adult males. ${ }^{30}$ This would suggest that dogs would proportionately accommodate larger cricothyrotomy tubes than a human of equivalent weight, and current human recommendations for maximal outer tube diameter of $8-9 \mathrm{~mm}^{7}$ may not apply to larger dogs. Due to the marked variation in small animal patient weight and size, the authors recommend attempting to place the largest tube, which would comfortably fit without excessive force. A tube size half of what is estimated for endotracheal intubation is a good starting point. ${ }^{5}$

The third objective was to assess the time taken for correct CTT tube placement using the modified technique. Successful CTT is defined not only by correct placement of the tube, but also placement within a defined time period. A time limit of between 180 and 300 seconds has been suggested as acceptable for a CTT procedure. ${ }^{9}$ In this study, there was marked variation in procedure time with a range of 35-240 seconds. It should be noted the quickest times of 40 seconds and 35 seconds were in the last three pigs and were both successfully placed on the first attempt. Pigs 2 through 6 required a more extensive approach with some degree of additional dissection or manipulation of equipment, which took a longer time. It was observed during the experiment that the operator was inadvertently grasping the CTT tube/urinary catheter apparatus by the tube itself. When introduced to the stab incision in this manner, the urinary catheter was pushed back into the tube and the benefit of the bougie/guidewire was lost. Placement times were improved by grasping the urinary catheter alone distal to the tube to ensure its steady advancement into the trachea. The proximal aspect of the catheter was subsequently grasped and held in a static position prior to feeding the CTT tube into the trachea.
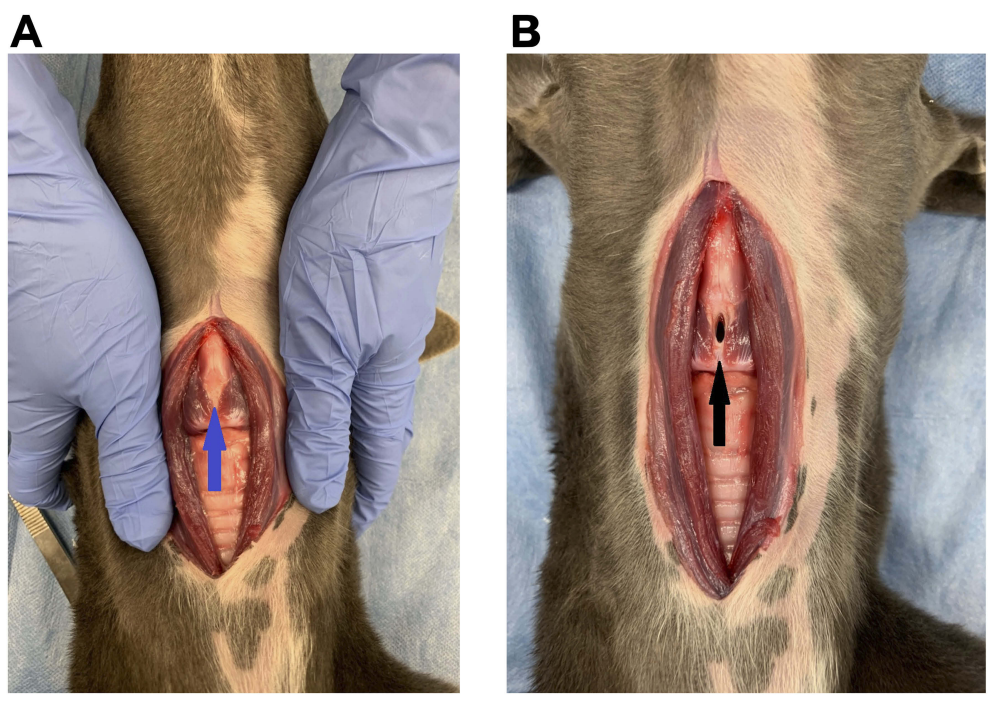

Figure 6 Dissection depicting the ventral laryngeal anatomy in a cadaver dog. (A) Blue arrow points to intact cricothyroid membrane and ligament. (B) Black arrow indicates the incision in the cricothyroid ligament. The cricothyroid membrane is located on the ventral aspect of the larynx, joining the caudoventral border of the thyroid cartilage and the cranioventral aspect of the cricoid cartilage. The medial part of the cricothyroid membrane is termed the cricothyroid ligament. The ligament is devoid of a major blood supply but may have small vessels associated near the cricoid and thyroid attachments. ${ }^{26}$ 
Another reason for the increased time taken is the larger skin to cricothyroid membrane distance encountered in pigs. Comparatively, these pigs would have been classed as an obese patient by human standards. Initiating the procedure with a wider exposure (Figure 4) rather than a stab incision may be indicated in such patients ${ }^{7}$ and could have improved placement accuracy and time.

Management of the cricothyrotomy tube after placement should be similar to tracheostomy tube care. Respiratory secretions will accumulate due to the lack of humidification and warming of the air as it bypasses the upper airway. ${ }^{27}$ Complete blockage or dislodgement of tracheostomy tubes is a known complication in veterinary patients and can be potentially life-threating. ${ }^{32}$ It is recommended that patients with cricothyrotomy tubes are continuously monitored for signs of tube obstruction. ${ }^{33}$ The intention of the CTT procedure described is rapid establishment of an airway to prevent death by asphyxiation in a CICO situation. If it is anticipated that the obstruction will remain for more than a few hours, the authors recommend to subsequently perform a standard surgical tracheostomy under controlled conditions, which may be easier to manage in the longer term. The wide approach and the presence of stay sutures around tracheal rings also allows the tracheostomy site to be easily located for tube changes. If rapid resolution of an obstruction is achievable, then the CTT tube should be removed. After removal of the tube, the stoma site is left open to heal by second intention.

An acknowledged limitation of this study is the use of pigs as an experimental model for small animals such as dogs and cats. A study comparing CTT tube placement between human and canine models showed marked variation in success due to the different anatomy between species. ${ }^{34}$ However, porcine models are widely used in human and veterinary medicine and have been specifically used for CTT simulations. ${ }^{25}$

The difference in laryngeal anatomy could have also explained the relative ease of tube placement in our dog cadaver models when compared to the pigs. A ventral deviation of the airway in pigs at the larynx has been well described in the veterinary literature compared with straighter airways in small animals. ${ }^{35,36}$ Furthermore, a prominent caudoventral border of the thyroid cartilage can be appreciated when comparing the pig larynx in Figure 4 to that of the dog in Figure 6. These factors may have potentially obstructed passage of the tube depending on the angle of insertion. When performing the procedure in dogs, the authors found that the caudal boder of the cricoid cartilage is far more defined than the pig. This also serves as an important surface landmark in the dog. Digital palpation from both the cranial and caudal aspects will assist in accurate identification of the cricothyroid notch. These anatomical variations as well as the relatively large soft tissue coverage described above could have been what caused some difficulty when working with the porcine model.

Ethical considerations also influenced the use of pigs for this live model. Pigs were re-used from a non-recovery veterinary student practical to ensure an accurate model for assessing ventilation without resulting in unnecessary discomfort or death of animals specifically for this project. The pigs would all have been humanely euthanized following the surgery practical regardless of our intervention.

Another limitation was the small number of animals examined. In addition, one veterinarian performed the technique for all pigs and so was able to become increasingly proficient. General practice veterinarians are likely seldom to perform such a technique on live patients.

A known limitation was the measurement of $\mathrm{ETCO}_{2}$ opposed to $\mathrm{PaCO}_{2}$ to assess ventilation. $\mathrm{ETCO}_{2}$ is known to underestimate $\mathrm{paCO}_{2}$ by approximately $2-6 \mathrm{mmHg}$. ${ }^{1}$ Although $\mathrm{ETCO}_{2}$ reflects $\mathrm{PaCO}_{2}$, increased $\mathrm{ETCO}_{2-}$ $\mathrm{PaCO}_{2}$ gradients can occur in conditions such as ventilation/perfusion (V/Q) mismatch which would result in reduced accuracy of data. It is possible that increased gradients may have occurred without the authors' knowledge.

This study was designed as a pilot study and proof of concept. A larger study comparing various surgical airway access techniques in more common species is recommended. Furthermore, a sustained period of monitoring, greater than 10 mins, may give a better indication of the longer-term sustainability of ventilation and whether respiratory fatigue due to tube resistance may ensue.

\section{Conclusion}

The modified laryngeal handshake and tube CTT technique detailed above demonstrated promise in this pilot study. It potentially fulfills the requirements of being rapid, easy to learn and uses commonly available equipment to veterinary general practitioners. Importantly, it permits ventilation and may represent a viable alternative to other established front of neck airway access techniques in small animal emergency medicine.

\section{Acknowledgments}

The authors would like to thank Dr Nicholas Cowling for his assistance in clinical anesthesia during the project. This research was supported by the University of Queensland, School of Veterinary Science. 


\section{Disclosure}

The authors declare no conflicts of interest in this work.

\section{References}

1. Silverstein DC, Hopper K, Silverstein DC. Small Animal Critical Care Medicine. 2nd ed. Saint Louis, Missouri: Elsevier; 2015.

2. Creedon JMB, Davis H. Advanced Monitoring and Procedures for Small Animal Emergency and Critical Care. Wiley-Blackwell; 2012.

3. Ziebart A, Garcia-Bardon A, Kamuf J, et al. Pulmonary effects of expiratory-assisted small-lumen ventilation during upper airway obstruction in pigs. Anaesthesia. 2015;70(10):1171-1179. doi:10.111 1/anae. 13154

4. Watson N, Canelli R, Zayaruzny M. A simple device constructed from easily accessible components improves emergency percutaneous transtracheal ventilation. Crit Care Med. 2012;40(12):U129U129. doi:10.1097/01.ccm.0000424677.97724.9b

5. Hansen IK, Eriksen T. Cricothyrotomy: possible first-choice emergency airway access for treatment of acute upper airway obstruction in dogs and cats. Vet Rec. 2014;174(1):17. doi:10.1136/vr.102286

6. Rehm CG, Wanek SM, Gagnon EB, Pearson SK, Mullins RJ. Cricothyroidotomy for elective airway management in critically ill trauma patients with technically challenging neck anatomy. Crit Care. 2002;6(6):531-535.

7. Onrubia X, Frova G, Sorbello M. Front of neck access to the airway: a narrative review. Trends Anaesth Crit Care. 2018;22:45-55. doi:10.1016/j.tacc.2018.06.001

8. Frerk C, Mitchell VS, McNarry AF, et al. Difficult airway society 2015 guidelines for management of unanticipated difficult intubation in adults. Br J Anaesth. 2015;115(6):827-848. doi:10.1093/bja/aev371

9. Langvad S, Hyldmo PK, Nakstad A, Vist G, Sandberg M. Emergency cricothyrotomy - a systematic review. Scand J Trauma Resusc Emerg Med. 2013;21(1):43. doi:10.1186/1757-7241-21-43

10. Romita MC, Colvin SB, Boyd AD. Cricothyroidotomy - its healing and complications. Surg Forum. 1977;28:174-175.

11. Koopmann CF, Feld RA, Coulthard SW. The effects of cricoid cartilage injury and antibiotics in cricothyroidotomy. $\mathrm{Am} J$ Otolaryngol. 1981;2(2):123-128.

12. Bjoraker GD, Kumar BN, Brown CDA. Evaluation of an emergency cricothyrotomy instrument. Crit Care Med. 1987;15(2):157-160. doi:10.1097/00003246-198702000-00016

13. Manczur PT, Greenough FA, Nicholson FG, Rafferty FG. Resistance of pediatric and neonatal endotracheal tubes: influence of flow rate, size, and shape. Crit Care Med. 2000;28(5):1595-1598. doi:10.1097/ 00003246-200005000-00056

14. Lumb AB. Nunn's Applied Respiratory Physiology EBook. Saintt Louis: Elsevier; 2016.

15. Coté JC, Eavey DR, Todres DI, Jones ED. Cricothyroid membrane puncture: oxygenation and ventilation in a dog model using an intravenous catheter. Crit Care Med. 1988;16(6):615-619.

16. Mazzaferro EM. Temporary tracheostomy. Top Companion Anim Med. 2013;28(3):74-78. doi:10.1053/j.tcam.2013.06.002

17. Baker PA, O'Sullivan EP, Kristensen MS, Lockey D. The great airway debate: is the scalpel mightier than the cannula? BJA. 2016;117 (supp11):i17-i19. doi:10.1093/bja/aew219

18. Greenland KB, Bradley WPL, Chapman GA, Goulding G, Irwin MG. Emergency front-of-neck access: scalpel or cannula - and the parable of Buridan's ass. BJA. 2017;118(6):811-814. doi:10.1093/bja/aex101
19. Varvinskiy A, Hinde T. 21st annual scientific meeting of the difficult airway society: lessons learned and glimpses of the future. $B J A$. 2017;119(3):345-347. doi:10.1093/bja/aex194

20. Boon JM, Abrahams PH, Meiring JH, Welch T. Cricothyroidotomy: a clinical anatomy review. Clin Anat. 2004;17(6):478-486. doi:10.1002/ ca.10231

21. Cook TM, Woodall N, Frerk C. Major complications of airway management in the UK: results of the fourth national audit project of the Royal College of Anaesthetists and the Difficult Airway Society. part 1: anaesthesia. Br J Anaesth. 2011;106(5):617-631. doi:10.1093/bja/aer058

22. Ravlo O, Bach V, Lybecker H, Møller JT, Werner M, Nielsen HK. A comparison between two emergency cricothyroidotomy instruments. Acta Anaesthesiol Scand. 1987;31(4):317-319.

23. Eisenburger LP, Laczika LK, List LM, et al. Comparison of conventional surgical versus Seldinger technique emergency cricothyrotomy performed by inexperienced clinicians. Anesthesiology. 2000;92(3):687-690. doi:10.1097/00000542-200 003000-00012

24. Takayesu J, Peak D, Stearns D. Cadaver-based training is superior to simulation training for cricothyrotomy and tube thoracostomy. Off $J$ Ital Soc Intern Med. 2017;12(1):99-102.

25. Sorbello M, Godoroja D, Margarson M. Front-of-neck access and bougie trapping. Anaesthesia. 2018;73:1165-1175. doi:10.1111/ anae. 14411

26. Evans HE, DeLahunta A, Miller ME. Miller's Anatomy of the Dog. 4th ed. St. Louis: Elsevier Health Sciences; 2013.

27. Johnston SA, Tobias KM. Veterinary Surgery: Small Animal. 2nd ed. St. Louis, Missouri: Elsevier; 2018.

28. Zikes C, McCarthy T. Bilateral ventriculocordectomy via ventral laryngotomy for idiopathic laryngeal paralysis in 88 dogs. $J \mathrm{Am}$ Anim Hosp Assoc. 2012;48(4):234-244. doi:10.5326/JAAHA-MS5751

29. Fossum TW. Small Animal Surgery. 4th ed. St. Louis, Mo, London: Mosby; 2013.

30. Tayama N, Kaga K, Chan RW, Titze IR. Geometric characterization of the laryngeal cartilage framework for the purpose of biomechanical modeling. Ann Otol Rhinol Laryngol. 2001;110(12):1154-1161. doi:10.1177/000348940111001213

31. Kim MJ, Hunter EJ, Titze IR. Comparison of human, canine, and ovine laryngeal dimensions. Ann Otol Rhinol Laryngol. 2004;113 (1):60-68. doi:10.1177/000348940411300114

32. Guenther-Yenke CL, Rozanski EA. Tracheostomy in cats: 23 cases (1998-2006)৯. J Feline Med Surg. 2007;9(6):451-457. doi:10.1016/j. jfms.2007.06.002

33. Wuestenberg K. Clinical Small Animal Care: Promoting Patient Health and Preventing Complications. Chichester, West Sussex, UK, Ames, Iowa: Wiley-Blackwell; 2012.

34. McCarthy MC, Ranzinger MR, Nolan DJ, Lambert CS, Castillo MH. Accuracy of cricothyroidotomy performed in canine and human cadaver models during surgical skills training. J Am Coll Surg. 2002;195(5):627. doi:10.1016/S1072-7515(02)01337-6

35. Grimm K. Comparative anesthesia and analgesia of ruminants and swine. In: Veterinary Anesthesia and Analgesia: the Fifth Edition of Lumb and Jones. 5 ed. Chichester, UK: John Wiley \& Sons; 2017:743-753.

36. Steinbacher R, Von Ritgen S, Moens Y. Laryngeal perforation during a standard intubation procedure in a pig. Lab Anim. 2012;46(3):261263. doi:10.1258/la.2012.012032 


\section{Publish your work in this journal}

Veterinary Medicine: Research and Reports is an international, peerreviewed, open access journal publishing original research, case reports, editorials, reviews and commentaries on all areas of veterinary medicine. The manuscript management system is completely online and includes a very quick and fair peer-review system. Visit http://www.dovepress.com/testimonials.php to read real quotes from published authors.

Submit your manuscript here: http://www.dovepress.com/veterinary-medicine-research-and-reports-journal 